\title{
Phytophotodermatitis from making sangria: a phototoxic reaction to lime and lemon juice
}

\section{Margaret Mioduszewski MD, Jennifer Beecker MD}

Competing interests: None declared.

This article has been peer reviewed.

The authors have obtained patient consent.

Affiliation: Division of Dermatology, University of Ottawa, Ottawa, Ont.

Correspondence to: Margaret Mioduszewski, mmioduszewski@toh.on.ca

CMAJ 2015. DOI:10.1503 /cmaj.140942

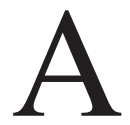
26-year-old woman presented to the emergency department with a painful blistering eruption on her hands. She had been squeezing limes and lemons while making sangria the previous day. She had spent the rest of the day outdoors in the sun without sunscreen. Hours later, she experienced a painful burning erythema followed by the formation of large, tense bullae on her hands (Figure 1). She was otherwise healthy and had no history of exposure to poison ivy or poison oak or to phototoxic drugs (e.g., naproxen and tetracycline), topical fragrances or other photosensitizing products that would make one consider the differential diagnosis of allergic contact dermatitis, drug-induced photosensitivity or pseudoporphyria. She had been seen in the emergency department on two previous occasions for similar presentations.

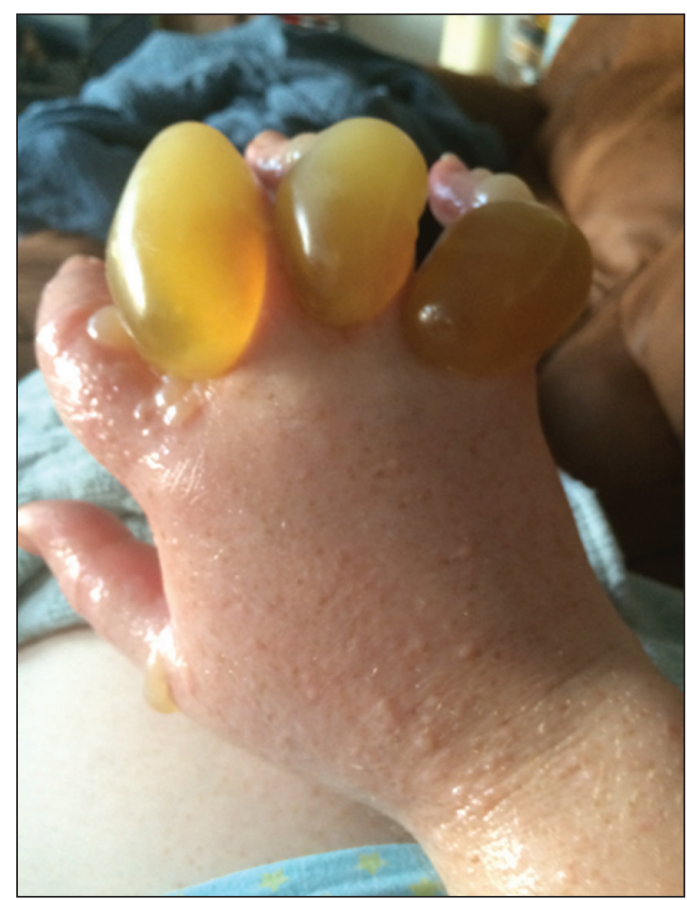

Figure 1: Large, tense unilocular bullae on the dorsal hand and interdigital spaces of a 26-year-old woman.
The patient had classic phytophotodermatitis, a phototoxic reaction to the limes and lemons in the sangria. The amount of sensitizing agent and exposure time determines the severity of the reaction. Previous sensitization is not necessary for the eruption to occur, because it is a nonimmunologic reaction, similar to a burn. Postinflammatory hyperpigmentation lasting weeks to months may develop. Management includes supportive treatment, wound care, trigger avoidance, patient education, and referral to a dermatologist if required.

Exposure to both ultraviolet light and lightsensitizing botanical substances in certain plants is required for phytophotodermatitis to develop. Erythema, bullae and eventual hyperpigmentation develop after skin becomes photosensitive due to contact with plants or fruits containing furocoumarins and is exposed to UVA radiation. ${ }^{1}$ The lesions can have bizarre shapes and are often painful, not pruritic, and appear only in sun-exposed areas, which helps differentiate the condition from plant-related contact dermatitis. ${ }^{2}$ Common culprits include citrus fruits (lime and lemon), parsnip, fennel, celery, parsley and giant hogweed. Phytophotodermatitis often occurs in the summer, but it can also develop during sun holidays in the winter: the so-called "Mexican beer (or margarita) dermatitis."

\section{References}

1. Shah N. What is this streaky rash? JAAPA 2014;27:18-9.

2. Bowers AG. Phytophotodermatitis. Am J Contact Dermat 1999; 10:89-93.

Clinical images are chosen because they are particularly intriguing, classic or dramatic. Submissions of clear, appropriately labelled high-resolution images must be accompanied by a figure caption and the patient's written consent for publication. A brief explanation (250 words maximum) of the educational significance of the images with minimal references is required. 